\title{
From Models to Languages
}

\section{Preface}

This special issue is devoted to various areas in Theoretical Computer Science. The issue took inspiration from the 13th Italian Conference on Theoretical Computer Science (ICTCS 2012), held at University of Insubria in Varese, Italy, on September 19-21 2012. The special issue contains seven papers, that have been originated from the work in progress presented at the conference, and that have been accepted for publication after a rigorous review process and revisions.

We kindly thank all the authors of the published papers, as well as all the participants to ICTCS 2012, who make it such an exciting event.

We are very grateful to the referees who devoted their precious time to produce thorough reviews. Their valuable comments and suggestions improved a lot the submitted manuscripts.

We are especially thankful to Professor Damian Niwinski, Editor-in-Chief of Fundamenta Informaticae, for accepting this special issue and for his help throughout the publication process.

Lastly, we wish to dedicate this special issue to the memory of Professor Alberto Bertoni, who was one of the co-founders of the Italian chapter of the European Association for Theoretical Computer Science, and passed away on February 2014.

\section{The Editors}

\section{Pierpaolo Degano}

Universita di Pisa

Juhani Karhumäki

University of Turku

Paolo Massazza

University of Insubria 